\title{
Self-Assembly of Organocyanide Dianions and Metal-Organic Macrocycles into Polymeric Architectures Including an Unprecedented Quadruple Helical Aperiodic Structure
}

Xuan Zhang, ${ }^{\dagger}$ Hanhua Zhao, ${ }^{\dagger}$ Lukas Palatinus, ${ }^{+}$Kevin Gagnon, ${ }^{\S}$ John Bacsa, ${ }^{\text {" Kim R. }}$ Dunbar*t

'Department of Chemistry, Texas A\&M University, College Station, TX 77842, USA

${ }^{*}$ Institute of Physics of the AS CR, v.v.i., Cukrovarnicka 10, 16200 Prague 6, Czech Republic

${ }^{\S}$ Advanced Light Source, Lawrence Berkeley National Lab, Berkeley, CA 94720, USA

"Department of Chemistry, Emory University, Atlanta, GA 30322, USA

\section{Electronic supporting information}




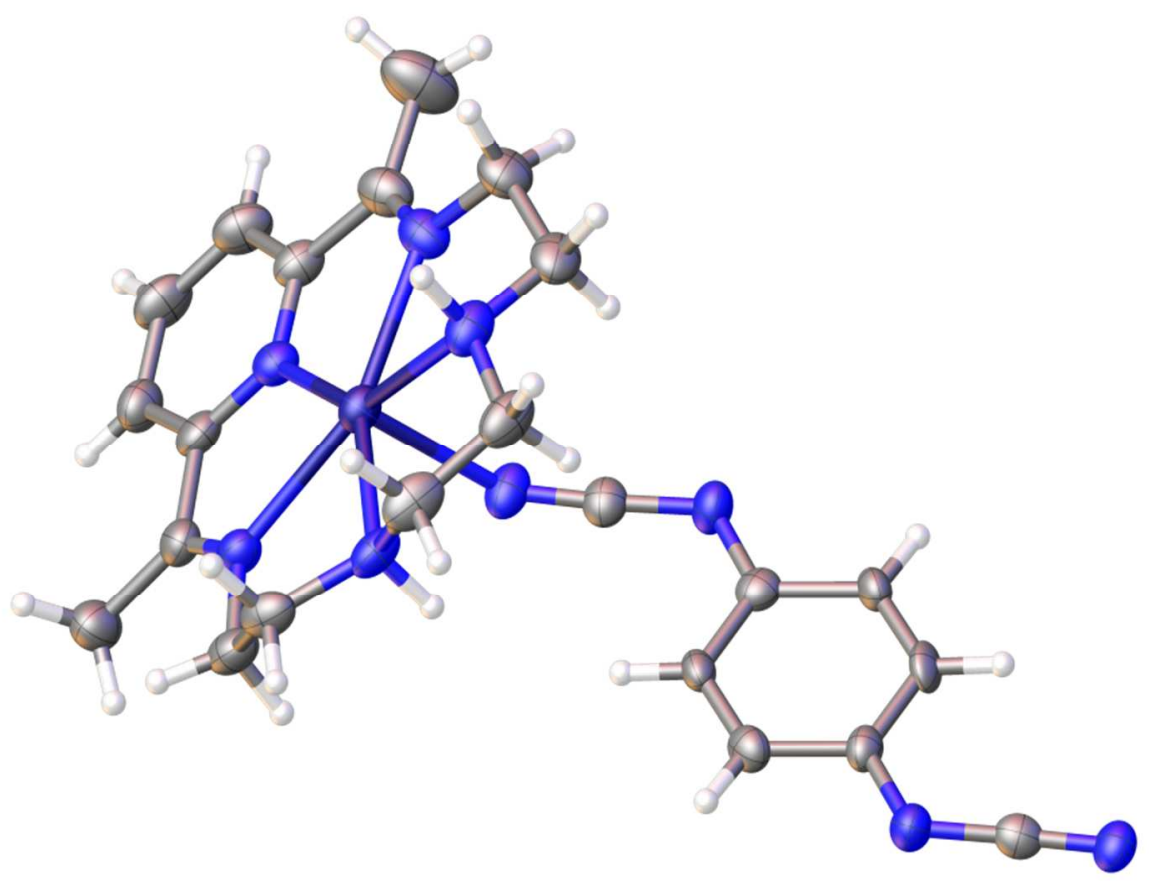

Figure S1. Asymmetric unit of compound 2. Thermal ellipsoids are drawn at the $50 \%$ level. The free solvent molecules are omitted for the sake of clarity. 


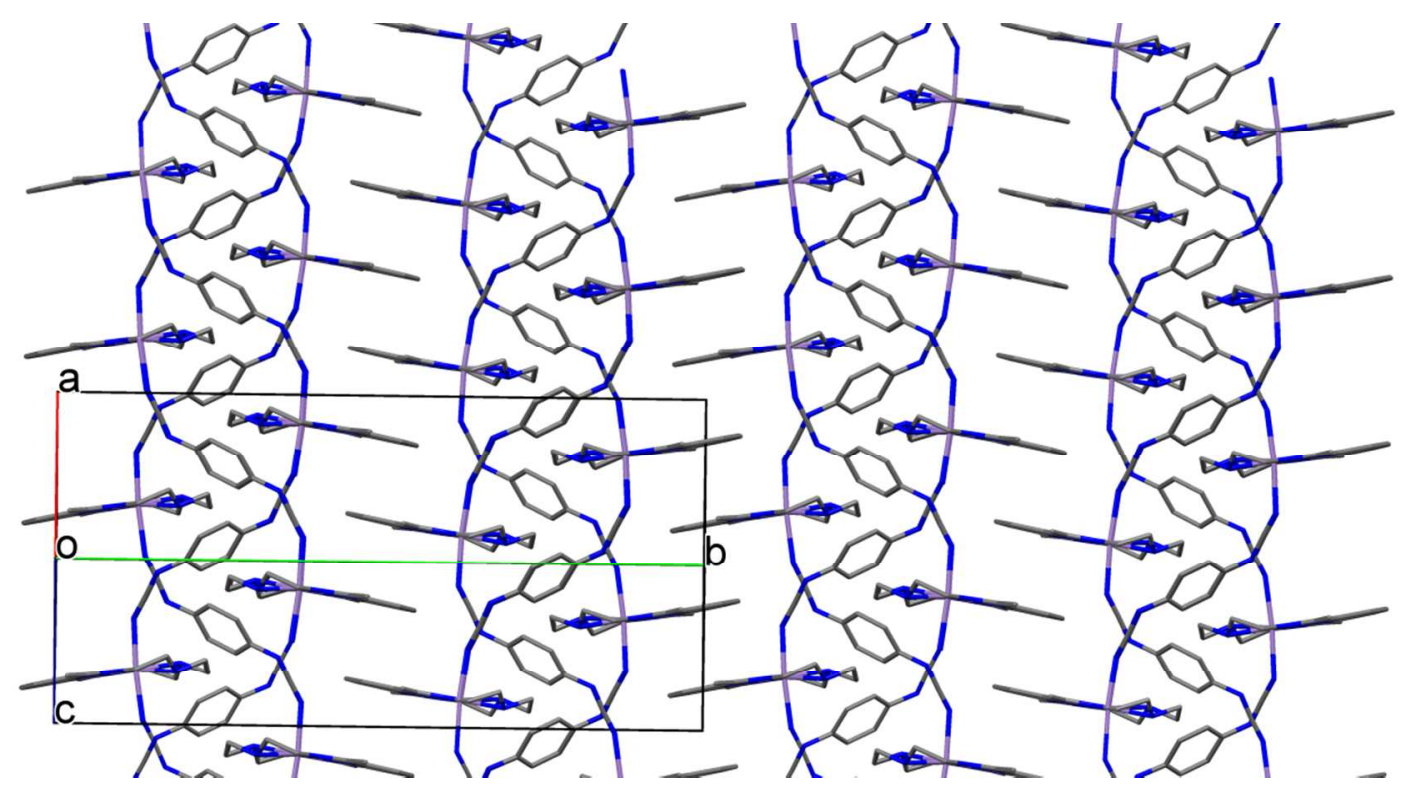

Figure S2. Packing diagram of compound $\mathbf{2}$ viewed along one of the ac diagonal direction to highlight the $\pi-\pi$ stacking interactions between the pyridyl groups of the dpop ligands. Solvent molecules in the channels along the a axis and hydrogen atoms are omitted for the sake of clarity. 


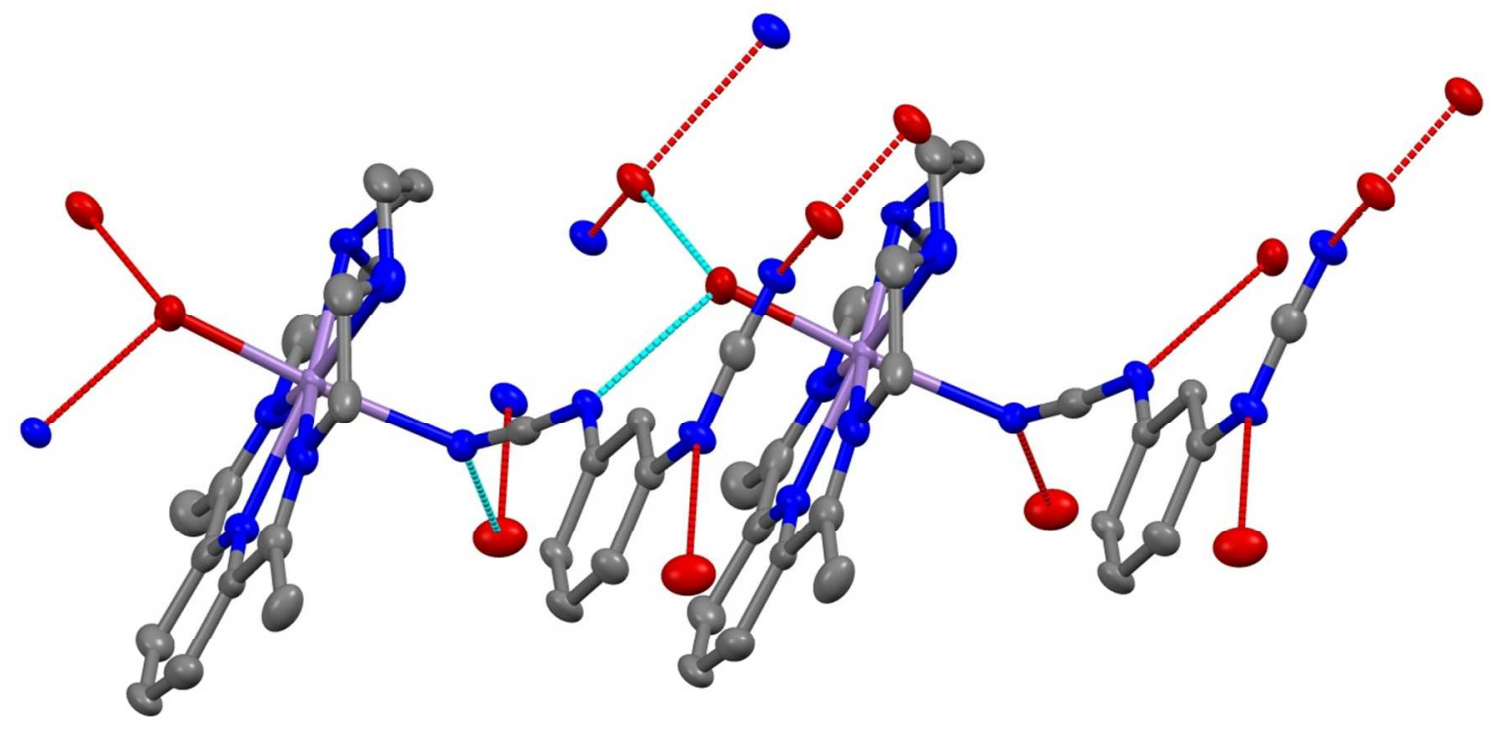

Figure S3. Crystal structure of compound $\mathbf{5}$ showing the hydrogen bonding interactions in the structure (dotted lines). Some molecules are shown as fragments with only the atoms involved in the hydrogen bonding interactions. Thermal ellipsoids are drawn at the $50 \%$ probability level. 